\title{
Demonstration of Machine Learning Capabilities on Internet of Things Devices
}

\author{
Abhinandan H. Patil \\ 14 Inc, Belgaum, Karnatka, India \\ Abhinandan_patil_1414@yahoo.com
}

\begin{abstract}
Since the problem definition mentioned in the title of this paper is very broad it was narrowed down to temperature sensing using the loT device and demonstrating the machine learning capabilities using the TensorFlow with the Python libraries. The data was started collecting starting 1:45 PM and collected till 6:00 PM. As the temperature in India starts cooling down from 2:00 till the evening, we should be getting down-ward slope i.e temperature starts tapering down. It is clearly linear regression problem where the slope is down-ward as we proceed further in time line. If we start collecting the data in the morning and collect till after-noon we should again get the linear regression model however this time the temperature increases as we proceed in the time line till 2:00 PM.
\end{abstract}

Keywords: Weka; Internet of Things; Machine Learning; Arduino; Python

\section{Introduction}

Following pre-requisites are needed for this experiment.

Table 1 Pre-requisities for the experiment

\begin{tabular}{|c|c|c|}
\hline Pre-requisites & Item name & Comments \\
\hline Operating System (Software) & Kubuntu/Windows & $\begin{array}{l}\text { As per the performance } \\
\text { requirements }\end{array}$ \\
\hline IDE(Software) & $\begin{array}{l}\text { Visual Studio } 2019 \text { and Arduino } \\
\text { Uno }\end{array}$ & $\begin{array}{l}\text { On Kubuntu bare-metal vi editor } \\
\text { along with command prompt } \\
\text { performs the job much faster }\end{array}$ \\
\hline Python libraries (Software) & $\begin{array}{l}\text { Python3, TensorFlow, matplotlib } \\
\text { and Tk }\end{array}$ & $\begin{array}{l}\text { Problem was attempted on } \\
\text { Kubuntu with the Java Standard } \\
\text { Edition (JSE) and with the limited } \\
\text { success }\end{array}$ \\
\hline Weka (Software) & Weka3.9 & Ease of using is much better. \\
\hline IoT device (Hardware) & Arduino Uno & $\begin{array}{l}\text { Available online at Amazon India } \\
\text { and far easy to use and }\end{array}$ \\
\hline
\end{tabular}




\begin{tabular}{|l|l|l|}
\hline & & $\begin{array}{l}\text { inexpensive compared with } \\
\text { raspberry pi }\end{array}$ \\
\hline Temperature Sensor (Hardware) & LM35 & Available online at Amazon India \\
\hline
\end{tabular}

\section{Temperature Sensor using the LM 35 Arduino Uno}

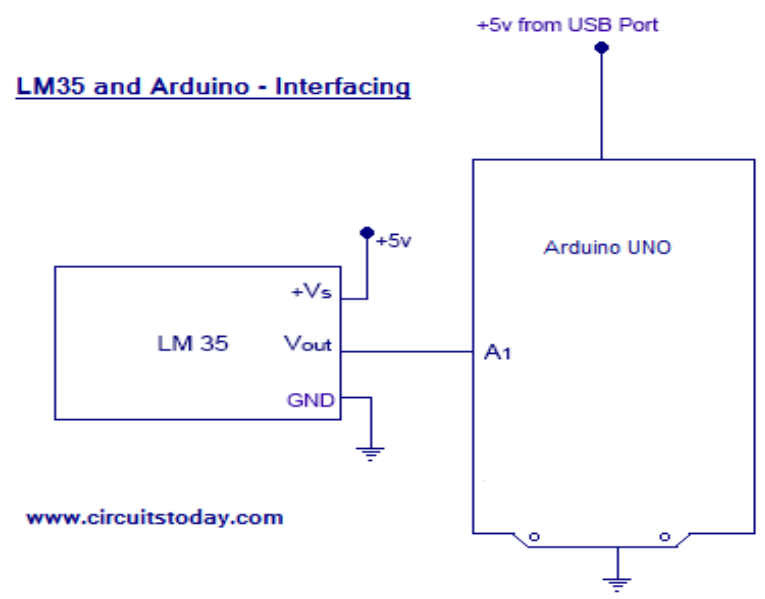

Figure 1. Circuit for LM 35

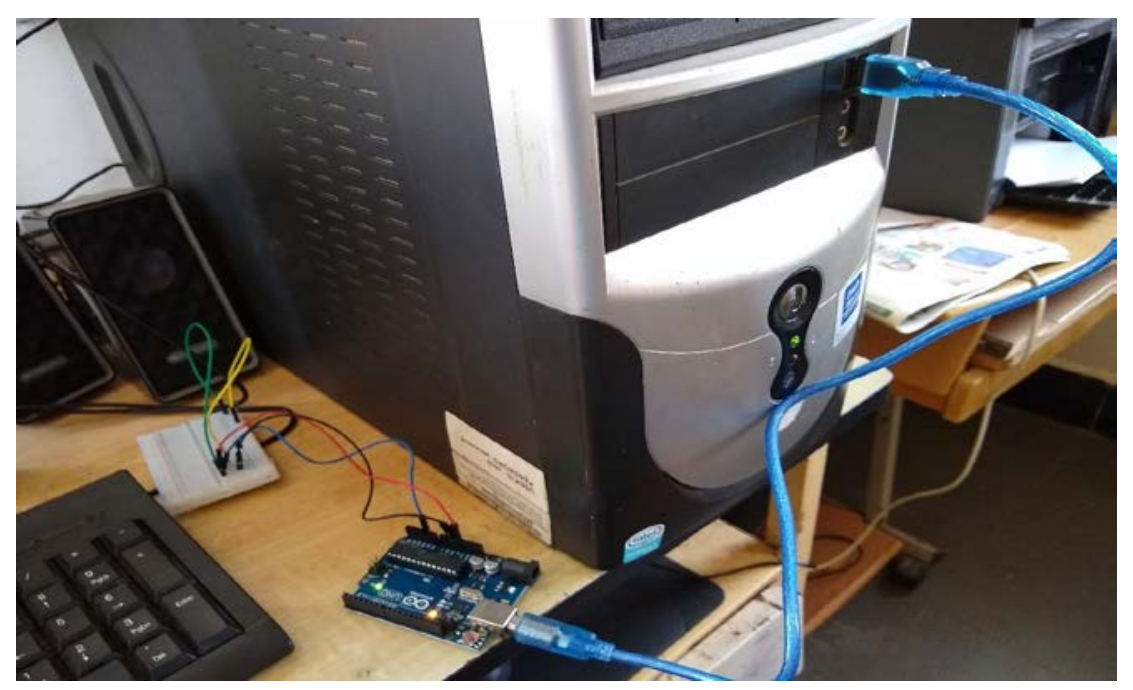

Figure 2. Arduino Interfaced with PC 


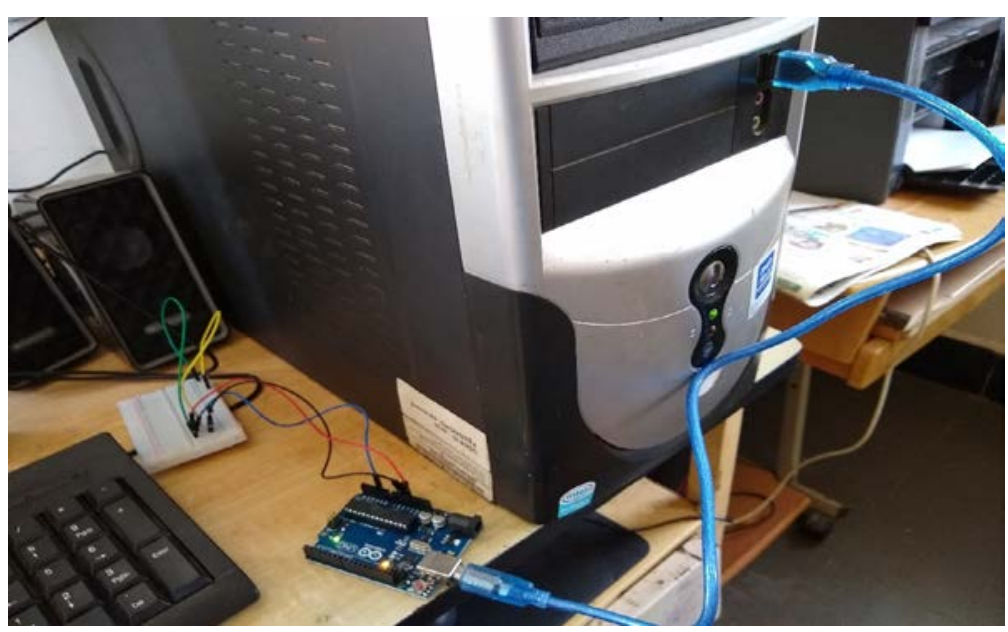

Figure 3. Arduino Intefaced with PC

\section{Experiment}

A First interface the sensor to Arduino board using the circuit as show above. Install the Arduino IDE. Then flex the following code to the microcontroller.

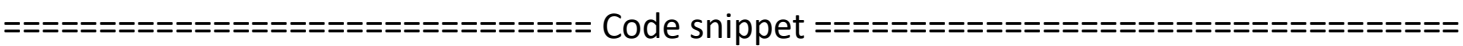

const int sensor=A1; // Assigning analog pin A1 to variable 'sensor'

float tempc; //variable to store temperature in degree Celsius

float tempf; //variable to store temperature in Fahreinheit

float vout; //temporary variable to hold sensor reading

void setup()

\{

pinMode(sensor,INPUT); // Configuring pin A1 as input

Serial.begin(9600);

\}

void loop()

\{

vout=analogRead(sensor);

vout $=($ vout $* 500) / 1023$;

tempc=vout; // Storing value in Degree Celsius

tempf=(vout*1.8)+32; // Converting to Fahrenheit

Serial.print("in DegreeC=");

Serial.print("\t"); 
Serial.print(tempc);

Serial.println();

Serial.print("in Fahrenheit=");

Serial.print("\t");

Serial.print(tempf);

Serial.println();

delay(60000); //Delay of 60 seconds for ease of viewing

\}

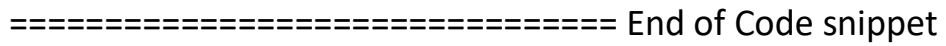

Using the COM3 port capture the reading (Arduino Uno IDE -> Tools -> Serial Monitor).

The raw data is transferred to the text file. The Extract transform operation is manual. The data is extracted such that only two parameters are present viz.

1. Time of data capture

2. Temperature in degree Centigrade.

Then use the following code [2] to model the data in a linear regression model. Visual studio 2019 was used as the Integrated Development environment.

=========================== Start of python ML Code snippet ==========================

II

A linear regression learning algorithm example using TensorFlow library.

Author: Aymeric Damien

Project: https://github.com/aymericdamien/TensorFlow-Examples/

II'

from_future_import print_function

import tensorflow as $\mathrm{tf}$

import numpy

import matplotlib.pyplot as plt

rng = numpy.random

\# Parameters

learning_rate $=0.01$

training_epochs $=1000$ 
display_step $=50$

\# Training Data

\# Change the following data as per the requirement

train_X

numpy.asarray([13.47,13.48,13.49,13.50,13.51,13.52,13.53,13.54,13.55,13.56,13.57,13.58,13.59,14.00, $14.01,14.02,14.03,14.04,14.05,14.06,14.07,14.08,14.09,14.10,14.11,14.12,14.13,14.14,14.15,14.16,14.1$ 7,14.18,14.19,14.20,14.21,14.22,14.23,14.24,14.25,14.26,14.27,14.28,14.29,14.30,14.31,14.32,14.33,14. $34,14.35,14.41,14.42,14.43,14.44,14.45,14.46,14.47,14.48,14.49,14.50,14.51,14.52,14.53,14.54,14.55,1$ $4.56,14.57,14.58,14.59,15.00,15.01,15.02,15.03,15.04,15.05,15.06,15.07,15.08,15.09,15.10,15.11,15.12$, $15.13,15.14,15.15,15.16,15.17,15.18,15.19,15.20,15.21,15.22,15.23,15.24,15.25,15.26,15.27,15.28,15.2$ $9,15.30,15.31,15.32,15.33,15.34,15.35,15.41,15.42,15.43,15.44,15.45,15.46,15.47,15.48,15.49,15.50,15$. $51,15.52,15.53,15.54,15.55,15.56,15.57,15.58,15.59,16.00,16.01,16.02,16.03,16.04,16.05,16.06,16.07,1$ $6.08,16.09,16.10,16.11,16.12,16.13,16.14,16.15,16.16,16.17,16.18,16.19,16.20,16.21,16.22,16.23,16.24$, $16.25,16.26,16.27,16.28,16.29,16.30,16.31,16.32,16.33,16.34,16.35,16.41,16.42,16.43,16.44,16.45,16.4$ $6,16.47,16.48,16.49,16.50,16.51,16.52,16.53,16.54,16.55,16.56,16.57,16.58,16.59,17.00,17.01,17.02,17$. $03,17.04,17.05,17.06,17.07,17.08,17.09,17.10,17.11,17.12,17.13,17.14,17.15,17.16,17.17,17.18,17.19,1$ 7.20,17.21,17.22,17.23,17.24,17.25,17.26,17.27,17.28,17.29,17.30,17.31,17.32,17.33,17.34,17.35,17.41, $17.42,17.43,17.44,17.45])$

train_Y

numpy.asarray([73.35,76.87,70.71,68.95,68.95,68.07,72.47,69.83,69.83,66.31,75.11,75.11,62.79,64.55, $64.55,75.99,62.79,75.11,68.07,71.59,64.55,75.11,66.31,75.11,71.59,61.91,59.27,72.47,72.47,61.91,74.2$ $3,70.71,65.43,62.79,72.47,63.67,70.71,67.19,73.35,61.91,68.07,72.47,72.47,72.47,73.35,71.59,70.71,75$. $99,60.15,72.47,68.95,76.87,71.59,75.99,68.95,69.83,68.95,73.35,60.15,73.35,73.35,75.11,67.19,62.79,7$ $0.71,68.07,63.67,68.95,76.87,75.11,75.11,72.47,68.95,69.83,76.87,70.71,75.99,75.11,63.67,72.47,59.27$, $75.99,57.51,71.59,73.35,58.39,63.67,66.31,75.99,72.47,73.35,68.07,57.51,72.47,68.95,76.87,75.99,67.1$ $9,61.91,69.83,64.55,58.39,75.99,70.71,76.87,64.55,61.03,70.71,76.87,76.87,61.91,72.47,72.47,70.71,61$. $03,73.35,61.03,69.83,68.07,76.87,68.07,60.15,61.91,64.55,67.19,64.55,65.43,61.91,61.03,70.71,61.03,6$ $8.07,76.87,75.99,65.43,75.11,74.23,68.95,76.87,70.71,59.27,60.15,75.99,72.47,72.47,67.19,69.83,68.07$, $69.83,62.79,69.83,75.99,69.83,72.47,72.47,72.47,73.35,75.11,70.71,68.95,61.03,61.03,71.59,70.71,71.5$ $9,68.95,66.31,61.03,75.11,73.35,70.71,73.35,61.03,72.47,72.47,71.59,68.95,72.47,67.19,74.23,72.47,69$. $83,70.71,69.83,72.47,61.03,71.59,63.67,67.19,75.11,69.83,71.59,68.95,71.59,74.23,60.15,59.27,62.79,7$ $0.71,72.47,69.83,69.83,70.71,70.71,70.71,70.71,69.83,68.07,66.31,73.35,61.03,72.47,75.11,68.95,74.23$, $69.83,69.83,68.07,70.71])$

n_samples $=$ train_X.shape[0]

\# tf Graph Input

$\mathrm{X}=$ tf.placeholder("float")

$\mathrm{Y}=$ tf.placeholder("float")

\# Set model weights 
W = tf.Variable(rng.randn(), name="weight")

$\mathrm{b}=\mathrm{tf}$. Variable(rng.randn(), name="bias")

\# Construct a linear model

pred $=$ tf.add $($ tf.multiply $(X, W), b)$

\# Mean squared error

cost $=$ tf.reduce_sum $($ tf.pow $($ pred-Y, 2$)) /\left(2 * n \_s a m p l e s\right)$

\# Gradient descent

\# Note, minimize() knows to modify $\mathrm{W}$ and $\mathrm{b}$ because Variable objects are trainable=True by default optimizer = tf.train.GradientDescentOptimizer(learning_rate).minimize(cost)

\# Initialize the variables (i.e. assign their default value)

init = tf.global_variables_initializer()

\section{\# Start training}

with tf.Session() as sess:

\# Run the initializer

sess.run(init)

\# Fit all training data

for epoch in range(training_epochs):

for $(x, y)$ in zip(train_X, train_Y):

sess.run(optimizer, feed_dict $=\{X: X, Y: y\}$ )

\# Display logs per epoch step

if $($ epoch +1$) \%$ display_step $==0$ :

$c=$ sess.run(cost, feed_dict $=\{X:$ train_X,Y:train_Y $\}$ )

print("Epoch:", '\%04d' \% (epoch+1), "cost=", "\{:.9f\}".format(c), ।

"W=", sess.run(W), "b=", sess.run(b)) 


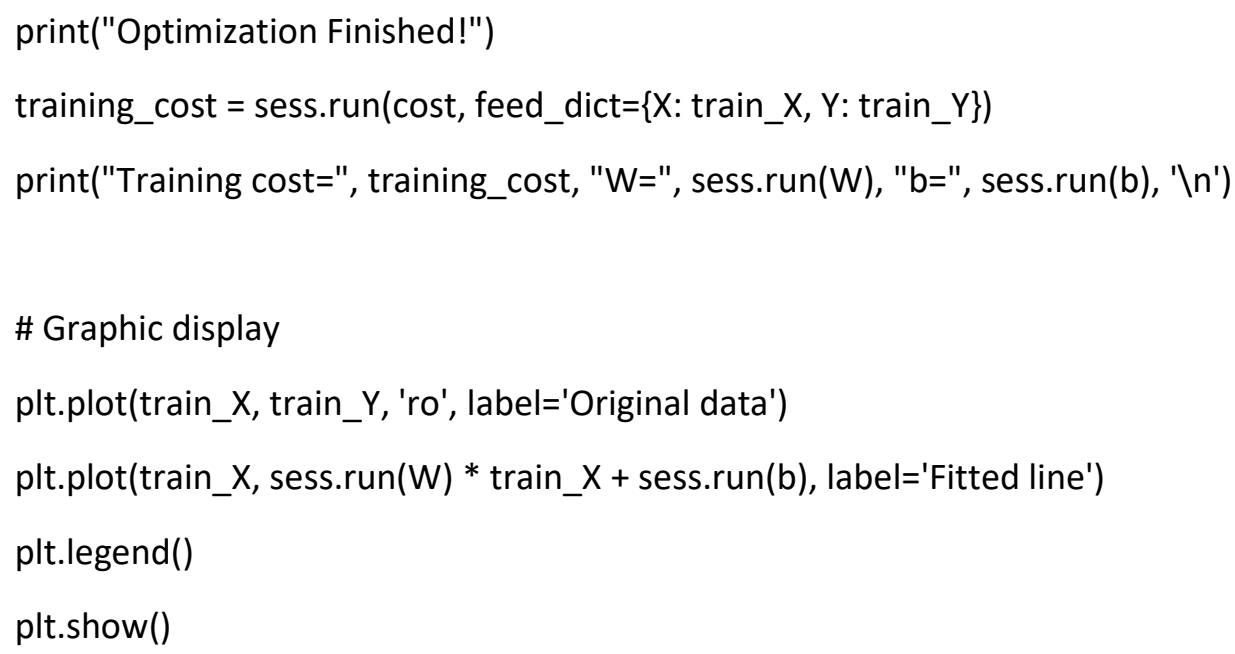

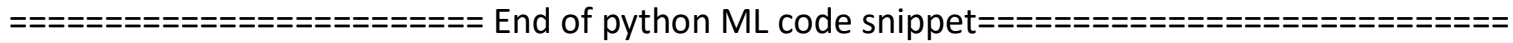

No changes were made to this code. Only data set was modified to suit the needs. However, python3, TensorFlow, matplotlib and tk libraries were installed to make the code work. The same thing was tried on Kbuntu 18.10 without IDE and performance was much superior. The mentioned job can be done with 
Weka as well [3]. The data was put in the comma separated value file. It was noticed that the Weka did the same job but with lot of ease.

\section{Results}

\subsection{TensorFlow Output}
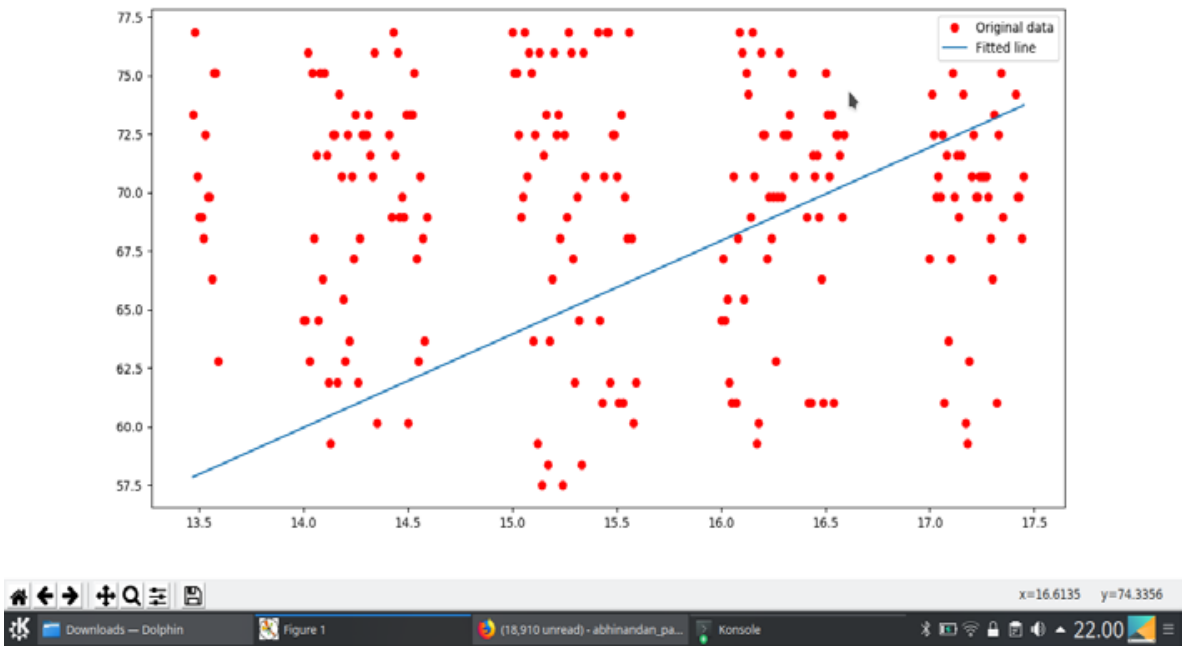

Figure 4. TensorFlow OutPut for Experiment

\subsection{Weka Output}

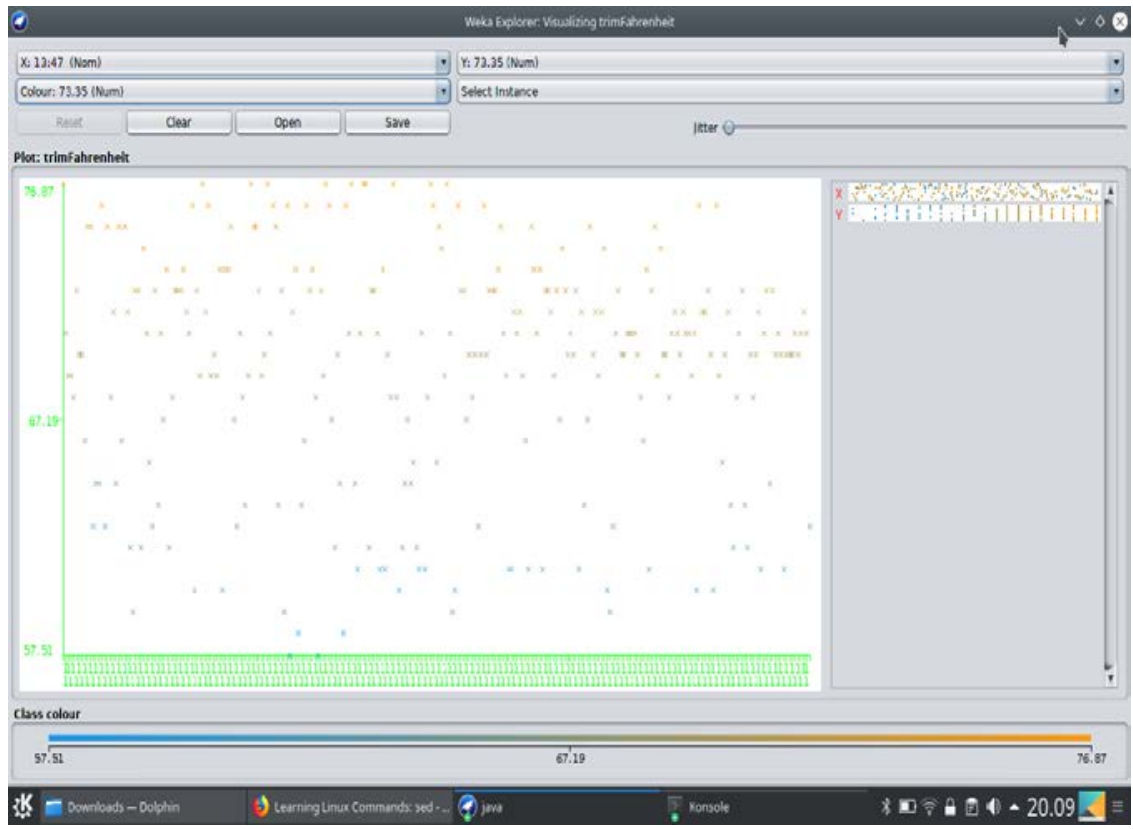

Figure 5. Weka OutPut for given Experiment 


\section{APPENDIX A}

Disclaimer: The temperature data was collected at Belgaum, Karnataka, India at room-temperature with ceiling fan on and may not be the exact readings. Further the data was collected using the USB port interfaced with the Windows 10 and Arduino IDE on COM3 port. The error rate was noticed to be high.

APPENDIX FOR ETL

Sample output from Arduino COM3 port:

13:47:36.282 $->$ in DegreeC $=\quad 22.97$

13:47:36.282 $->$ in Fahrenheit $=73.35$

13:48:36.290 $->$ in DegreeC $=24.93$

13:48:36.324 $->$ in Fahrenheit $=76.87$

13:49:36.326 $->$ in DegreeC $=21.51$

13:49:36.326 $->$ in Fahrenheit $=70.71$

13:50:36.311 $->$ in DegreeC $=20.53$

13:50:36.345 $->$ in Fahrenheit $=68.95$

13:51:36.335 $->$ in DegreeC $=\quad 20.53$

13:51:36.335 $->$ in Fahrenheit $=68.95$

13:52:36.347 $->$ in DegreeC $=20.04$

13:52:36.347 $->$ in Fahrenheit $=68.07$

13:53:36.344 $->$ in DegreeC $=\quad 22.48$

13:53:36.378 $->$ in Fahrenheit $=72.47$

13:54:36.356 $->$ in DegreeC $=\quad 21.02$

13:54:36.389 $->$ in Fahrenheit $=69.83$

13:55:36.368 $->$ in DegreeC $=21.02$

13:55:36.402 $->$ in Fahrenheit $=69.83$

13:56:36.392 $->$ in DegreeC $=19.06$

13:56:36.392 $->$ in Fahrenheit $=66.31$

13:57:36.403 $->$ in DegreeC $=23.95$

13:57:36.403 $->$ in Fahrenheit $=75.11$

1) cat veryimpdump.txt | awk '/Fahrenheit/ \{print\}' | sed 's/-> in Fahrenheit=/,/g' > Fahrenheit.txt

2) cat veryimpdump.txt | awk '/DegreeC/ \{print\}'| sed 's/-> in DegreeC=/,/g' > DegreeC.txt

3) cat DegreeC.txt | sed 's/.[0-9]\\{3\\}//g' 
Abhinandan H. Patil; Demonstration of Machine Learning Capabilities on Internet of Things Devices. Transactions on Machine Learning and Artificial Intelligence, Volume 7 No 2 April (2019); pp: 63-72

4) cat Fahrenheit.txt | sed 's/.[0-9]\\{3\\}//g'

5) Manual process to remove insignificant time lines

6) cat afterhours.txt | awk '/Fahrenheit/ \{print\}'| sed 's/-> in Fahrenheit=/,/g'| sed 's/.[0-9]\\{3\\}//g'

7) cat train.csv | awk '\{print \$1 \$2\}'| grep -v '^\$' | paste -s -d"," | sed 's/,,/,/g'

8) cat train.csv | awk '\{print \$3\}' | sed -e :a -e 'N;s/nn/,/;ba'

\section{REFERENCES}

[1] CircuitsToday.2019. CircuitsToday. Retrieved from CircuitsToday: http://www.circuitstoday.com/Im35-and-arduino-interfacing

[2] Daimen, A. 2019. Americ. From Americ: https://github.com/aymericdamien/TensorFlowExamples

[3] Edu, K. 2019. Kean Edu. From Kean Edu: https://www.kean.edu/ fosborne/bstat/09rc.html

[4] Weka. 2019. University of Waikato. From University of Waikato: https://www.cs.waikato.ac.nz/ml/weka/ 